\title{
An Aging Cyclists Time Trial Performances over Four Decades: A Case Study
}

\author{
Bryce Dyer
}

Bournemouth University

* Correspondence: Bryce Dyer. brdyer@bournemouth.ac.uk

Received: 15 June 2020; Accepted: 30 September 2020; Published: 30 September 2020

\begin{abstract}
: previous research has often highlighted the physiological decline an athlete will be subjected to as they age. However, whilst some studies have evaluated a large sample of athletes at a given age, few studies have evaluated a single athlete over a much longer period of time in sports such as cycling. This study assessed the time trial performances of a multiple national record holding male amateur cyclist from when they were aged between 37 to 75 years of age. 488 of their individual performances over nearly four decades were contrasted against a statistically generated baseline of athletes that they competed against during these events. The results indicated a relatively stable level of performance from aged 37-52 years of age. However, a noticeable decline began to take place at aged 61 which then degraded sharply at aged 70. Interestingly, the athlete did not exhibit a permanent reduction in their average velocity in their best $16.1 \mathrm{~km}$ and $40.2 \mathrm{~km}$ time trial performances until aged 70 . This suggests that despite the physiological decline that will eventually reduce a riders competitiveness, this case study demonstrated that it is feasible to continue the pursuit of personal records until relatively late in life.
\end{abstract}

Keywords: cycling; masters athlete; performance analysis; time trialling

\section{Introduction}

Within competitive cycling, a unique discipline is the 'individual time trial'. This cycling discipline requires a rider to cover a fixed distance at their highest obtainable average velocity. To achieve this requires the highest possible physiologically generated power (Jeukendrup et al. 2000), the optimised reduction of the aerodynamic drag of the rider and bicycle (Lukes et al. 2005) and the maximised mechanical efficiency of the cyclist's drivetrain (Zamparo et al. 2002). Insofar as the athletes who may compete in cycling time trials, 'masters athletes' are typically regarded as being older than 35 years of age and compete in organized forms of sport for older adults (Reaburn et al.
2008). An age-related decline in performance by masters athletes are well cited and have been observed across several endurance sports (Reaburn et al. 2008) including cycling upto 60 years of age (Ransdell et al. 2009) and across a variety of age groups (Peiffer 2008). These declines have been reported as curvilinear from age 35 years until approximately age $60-70$ years. The trend then changes to those that are negatively exponential thereafter (Reaburn et al. 2008). Whilst comparing a group of athletes at a range of ages is a typical approach to illustrate an age-based decline in performance, there is also value from obtaining longitudinal studies of specific athletes (Rathwell \& Young 2015) or rider case studies over large periods of time between formal testing (Mujika 2012) despite

(C) 2020 Fennell, licensee JSC. This is an Open Access article distributed under the terms of the Creative Commons Attribution License ((http://creativecommons.org/licenses/by/4.0/) which permits unrestricted use, distribution, and reproduction in any medium, provided the original work is properly cited. 
these being rarely investigated. This may be pertinent when it is considered that master's competitions are actually seeing a greater source of performance improvement than those of elite athletes in their prime (Akkari et al. 2015). As a result, this may suggest that future findings in this field may revise the conclusions of older studies. This case study will evaluate the performance of a time-trial cyclist over a relatively long period of time.

\section{Materials and Methods}

\section{Subjects}

A male cyclist acted as the basis for this case study. The subject was geographically located in the UK. The subject was 76 years of age with four decades of consistent competitive experience in UK-based cycling time trials. They were defined as an 'amateur cyclist' in that they did not use competitive cycling as a means of employment or income and would race throughout a calendar year at events of their choice and preference. The subjects' perceived standard as a racing cyclist was judged as high based upon them holding several national age group records held between the ages of 68-74 for the formal 10 mile $(16.1 \mathrm{~km})$ or 25 mile $(40.2 \mathrm{~km})$ time trial distances in the UK. The formal race distances are defined in miles in the UK but will be expressed in kilometres for the purposes of this paper.

Within the UK, the consistent format of competitive cycling time trials involves riders competing individually over several fixed race distances of 10-100 miles in length or using time constrained formats of 12 and 24 hours in duration (www.cyclingtimetrials.org.uk). Whilst the distance or duration remains the same, the race environment itself that a UK-based time trial cyclist will race over is an open environment. This means any performance could be influenced by external factors such as weather, road surface condition, gradients and the influence of any passing motor vehicle traffic. However, whilst these conditions are not standardised or definable per se', their philosophical influence have remained consistent in principle since the sports inception. This particular sporting environment has seen performance analysis applied to it previously (Dyer et al. 2016).

\section{Methodology}

The subject's results in cycling time trials from 1980-2020 were used as the basis of this analysis. The results were sourced from the time trialling governing body's web page for this participant's geographical home region (www.southdc.org.uk). The inclusion criteria of the participant's results were deemed that of any race that was potentially open to any competitors, irrespective of gender or age and was not that of a team based event such as a team time trial. Finally, the events analysed only comprised those that were competed over a fixed distance but not those of a fixed duration. This study obtained institutional ethical approval, informed consent from the participant and the results used for this analysis existed in the public domain.

\section{Statistical Analysis}

The participant's time trial results were statistically compared to that of an established baseline to assess the positive or negative changes in their performance. Traditionally, this can be achieved by comparing an athlete's performance to world or national records (Ransdell et al. 2009) or the use of metrics such as the riders power output. However, the ability to consider age relies on national age-related records being known retrospectively at the time the participant competed in each of their events but these were not available. Secondly, the means to record power 'in the field' by cyclists was not feasible four decades ago. Instead, the mean average of the ten fastest finishers of each event was utilised to provide a statistically calculated sociological baseline. A similar approach has been previously used to compare general athletic performance of an event against its medal podium (Dyer et al. 2015). To then compare the participants, the Performance Improvement Index (PII) has been used as a means to compare cycling performance (Haake 2009). The PII primarily assesses the change in performance from one data point to another. When considering timed events 
such as cycling time trials, Haake defines the PII as: $P I I=\left[\left(\frac{t_{1}}{t_{2}}\right)^{2}-\mathbf{1}\right] \times \mathbf{1 0 0}$

For this study, the PII between the mean of the 10 fastest riders (t1) and the participant (t2) was calculated for each individual event. This was deemed the Relative Performance Improvement Index (RPII). Additionally, to account for any changes the participant may have made in their training, event emphasis or technology, the mean of the best six RPII results from each year were also selected for analysis, with any years with less than six events then discarded.

To help ascertain what consistency existed in the improvements or decline in the participants' performance, the RPII of the best annually achieved $16.1 \mathrm{~km}$ and $40.2 \mathrm{~km}$ race distances was checked for statistical significance using a students paired t-test $(\rho<0.05)$.

\section{Results}

The participant completed 488 eligible time trial events during the time period of 19812019. The participant's results of their RPII from 37-75 years of age are illustrated in figure 1 .

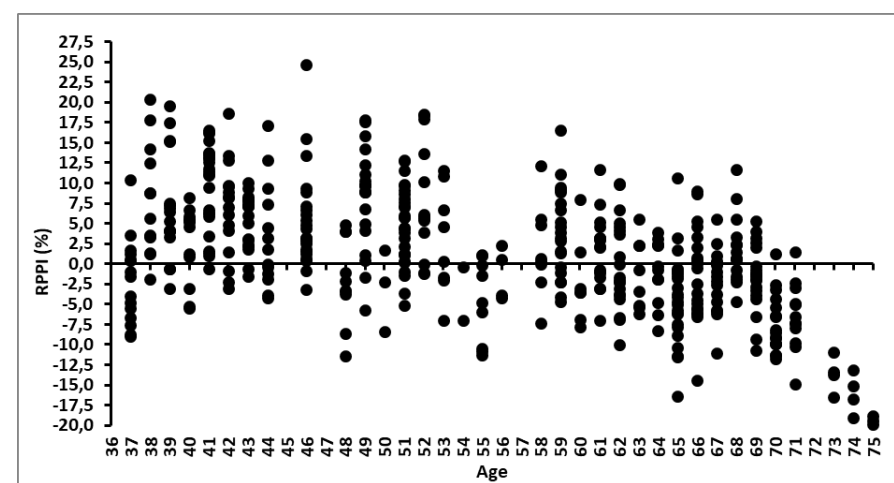

Figure 1. Relative Performance Improvement Index (RPII) change of participant aged 3775.

The participant experienced a noticeable decline in their performance over the evaluated time period. The participant remained positively competitive vs those in 1st-10th until approximately 52 years of age. The participant then seemed to undertake a reduced level of competitive participation and performance in the sport from ages 5457. From ages 58-68 their performance seemed to return to a positive level but lower than that of when 37-52 years of age. From the age of 65 , their performance level indicates a shift to being predominantly negative yet relatively stable until then degrading markedly from age 70 onwards. The six best RPII annual performances are shown in figure 2.

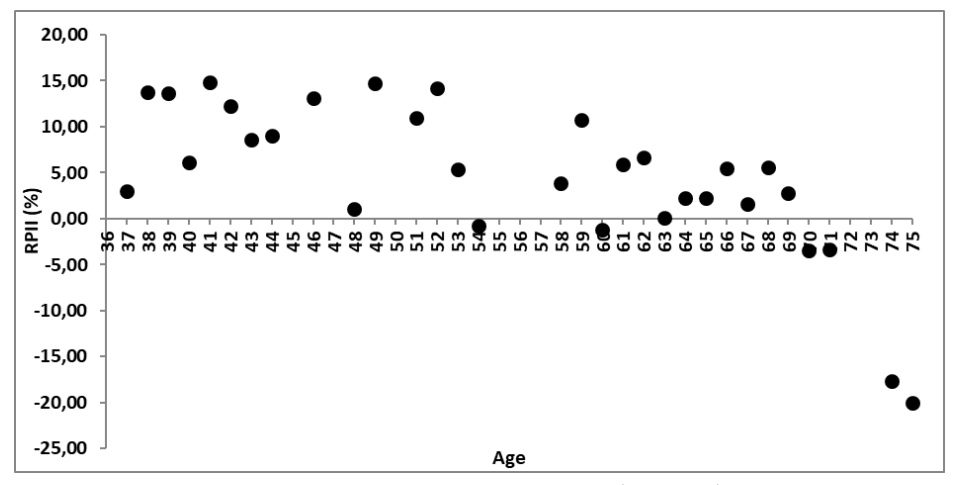

Figure 2. Best six annual Relative Performance Improvement Index (RPII) scores.

Figure 2 shows a reduced, yet still positive RPII from 52 years of age. After a short period of low or no race participation, it shows a progressively reducing RPII from age 58-69 years of age. The shift to a permanently negative level of performance is seen at age 70-74.

The participant's best annual performance over the $16.1 \mathrm{~km}$ racing distance is shown in figure 3. In this, their best average velocity achieved at each age is shown against the RPII with a 6-point polynomial line of best fit added to both traces.

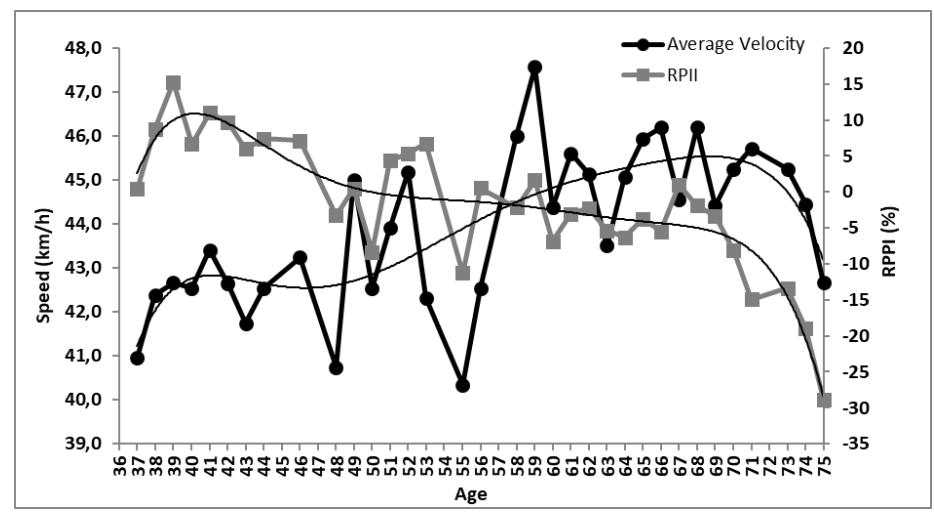

Figure 3. Best annual $16.1 \mathrm{~km}$ Relative Performance Improvement Index (RPII) score. 
In figure 3, the participant sees a general increase in race-based average velocity until 70 years of age before a noticeable decline takes place. Conversely, the RPII is in a generally progressive decline demonstrating a reduction in the participant's competitivity. The obtained velocity of the participant's age over the $40.2 \mathrm{~km}$ race distance is shown in figure 4.

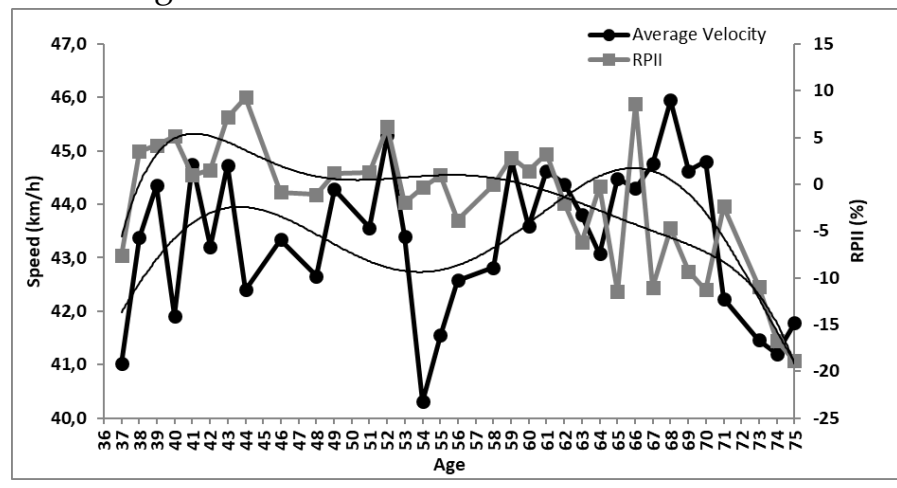

Figure 4. Best annual $40.2 \mathrm{~km}$ Relative Performance Improvement Index (RPII) score.

In figure 4, the decline in their performance, exhibited by the RPII, shifted progressively negative from 70 years of age. However, the participant was able to obtain average velocities that were in the range of 44$45 \mathrm{~km} / \mathrm{h}$ from age 36 up to age 70 and even exceeded this at age 68 .

Finally, both the $16.1 \mathrm{~km}$ and $40.2 \mathrm{~km}$ best annual performance RPII's shown in figures 3 and 4 were not significant from each other $(\mathrm{p}=0.9)$.

\section{Discussion}

In the case of this study, as expected, the participant has experienced a noticeable decline in their performance. However, they remained competitive with their performances remaining relatively stable and undiminished until approximately 52 years of age. It has been stated that whilst cycling performance does progressively decline, it can be well maintained in master's competitions until their late 60s (Baker \& Tang 2010). Aside from a period of reduced competition from when the participant was aged 54-57, that observation was supported by this case study. The clear negative degradation in performance took place from age 70 onwards which supports a general commentary on ageing time triallists made by Davison (2012 pg 234). It is conceded that the causes of the decline in the subject's performance post 70 years of age are likely to be complex and could equally be caused by social, economic, and lifestyle factors rather than purely that of their physiological degradation as well as a reduction in both training and competition. These potential issues are a limitation of this case study. Such confounding factors have also been conceded in master's studies before (Baker \& Tang 2010). However, it should be noted that the participant won and held the age-based national records over a variety of race distances at age 68-74 (https://www.vtta.org.uk/records) whilst this decline was taking place. This infers that the participant was likely intending to be competitive at this point, despite an obvious decline in their performance.

Figure 1 illustrated several occasions whereby the participant achieved a year possessing mainly negative results that were then followed by a return to better performances in ensuing years. The participant suggested these were due to changes in training methodology or other sociological factors. Whilst it is well cited that age-related performance-based decline is inevitable, the multi-faceted nature of performance cycling indicated in the introduction of this paper does mean that such decline was slowed or even temporarily reversed by the participants' interventions.

The PII has been successfully used to detect changes in sports technology (Haake 2009). In the case of time trialling, a notable innovation was the introduction of 'tri bars' during the 1980's. These changed the traditional method of riding a bicycle with relatively wide handlebars to assuming more of a 'tuck' with the hands positioned together and in front of the rider. This innovation saves rider energy at the same speed or increases their velocity for the same energy output (Sheel et al. 1996). It should be noted that the participant confirmed that they started using these in 1986 but none of the graphs showed a noticeable increase in race 
average velocity around this time. The reason for this only highlights the confounding variables such as weather or traffic conditions when competing in an open environment. As a result, the trends in this case study should be considered more important than any specific absolute values.

The result of the t-test suggests that their performance relative to their peers in both the $16.1 \mathrm{~km}$ and $40.2 \mathrm{~km}$ best annual performances were not significant from each other, irrespective of these different race distances. This suggests that any year to year RPII changes were unilateral to the athlete and not event specific when considering race durations ranging from 20 mins to circa one hour. When considering the participants best annual results of the $16.1 \mathrm{~km}$ and $40.2 \mathrm{~km}$ race distances, the participant surprisingly achieved some of their highest average velocities as they aged into their $60^{\prime}$ s, yet past the point where their RPII had already shown degradation. This could have been due to technological advancement, environmental changes (such as more favourable courses in terms of topography), atmospheric-based decreases in aerodynamic drag, environmental changes (such as changes in passing traffic levels) or combinations of these thereof. Thereby it is conceded that there is plenty of scope for random or confounding factors in cycling performance. Nonetheless, the perceived success on time trial cycling by its athletes could be judged in two different ways. If the goal is to remain competitive against other participants, there is obviously a point where this will degrade and that this case study fell broadly in line with previous research and a subsequent sharp loss of performance at aged 70 (Reaburn et al. 2008). However, if the primary aim is their pursuit of the highest possible average velocity they can achieve, this may still be achievable at a later point in life than the physiological decline alone has previously suggested. Provided the athlete is aware of the composite relationship between their training, equipment and environmental factors, they may be able to stimulate, slow down or even improve their personal level of performance. Since many master athletes may seek a personal record as a priority, this outcome could be seen favourably.

\section{Limitations}

There are two main limitations in this case study. The first is that the physiological and performance characteristics of the participant were not stated in the paper and would have likely changed over time. These would have included aspects such as their power output, VO2 max, body-mass and overall aerodynamic drag. However, the technology to record these was not available, not known or not feasible over the four decade time period.

Secondly, the use of a statistically generated baseline to compare the participant against does not account for any sociological changes that may have occurred in the sport over four decades. These could include changes in the sports general performances, or participation levels of this cycling discipline as well as specific riders entering or leaving the sport.

\section{Conclusion.}

This paper provided more evidence of the known physiological decline that takes place by masters cyclists in the form of a four decade-long case study. Despite this decline, this case study has shown how late in life a good level of relative performance was held and this may provide a useful case study for coaches and practitioners alike to note. Furthermore, it is also worth considering that athletes and coaches may be able to orchestrate a temporary reduction in this decline provided they remain sensitive and proactive in their awareness and manipulation of the other performance factors that occur in cycling time trials besides just that of the riders' physiology.

\section{Funding}

This research received no external funding 


\section{Acknowledgments}

The participant in particular is thanked for being willing to share their anecdotal experiences of their competitive endeavours.

\section{Conflicts of Interest}

The author declares no conflict of interest.

\section{References}

1. Akkari, A., Machin, D., \& Tanaka, H. (2015). Greater progression of athletic performance in older Masters athletes. Age and Ageing, 44(4), 683-686.

2. Baker, A. B., \& Tang, Y. Q. (2010). Aging performance for masters records in athletics, swimming, rowing, cycling, triathlon, and weightlifting. Experimental Aging Research, 36(4), 453-477.

3. Davison, R. (2012). The ageing cyclist, In Hopker, J., \& Jobson, S (Eds.). Performance Cycling: The Science of Success. A\&C Black.

4. Dyer, B., Hassani, H., \& Shadi, M. (2016). The analysis and forecasting of male cycling time trial records established within England and Wales. Journal of Sports Sciences, 34(13), 12221230.

5. Dyer, B. (2015). The progression of male 100 m sprinting with a lower-Limb amputation 1976-2012. Sports, 3(1), 30-39.

6. Haake, S. J. (2009). The impact of technology on sporting performance in Olympic sports. Journal of Sports Sciences, 27(13), 14211431.

7. Jeukendrup, A. E., Craig, N. P., \& Hawley, J. A. (2000). The bioenergetics of world class cycling. Journal of Science and Medicine in Sport, 3(4), 414-433.

8. Lukes, R. A., Chin, S. B., \& Haake, S. J. (2005). The understanding and development of cycling Engineering, 8(2), 59-74.

9. Ransdell, L. B., Vener, J., \& Huberty, J. (2009). Masters athletes: an analysis of running, swimming and cycling performance by age and gender. Journal of Exercise Science $\mathcal{E}$ Fitness, 7(2), S61-S73.

10. Peiffer, J. J., Abbiss, C. R., Chapman, D., Laursen, P. B., \& Parker, D. L. (2008). Physiological characteristics of masters-level cyclists. The Journal of Strength $\mathcal{E}$ Conditioning Research, 22(5), 1434-1440.

11. Mujika, I. (2012). The cycling physiology of Miguel Indurain 14 years after retirement. International Journal of Sports Physiology and Performance, 7(4), 397-400.

12. Rathwell, S., \& Young, B. W. (2015). Modelling commitment and compensation: A case study of a 52-year-old masters athlete. Qualitative Research in Sport, Exercise and Health, 7(5), 718-738.

13. Reaburn, P., \& Dascombe, B. (2008). Endurance performance in masters athletes. European Review of Aging and Physical Activity, 5(1), 31.

14. Sheel, A. W., Lama, I., Potvin, P., Coutts, K. D., \& McKenzie, D. C. (1996). Comparison of aero-bars versus traditional cycling postures on physiological parameters during submaximal cycling. Canadian Journal of Applied Physiology, 21(1), 16-22.

15. Zamparo, P., Minetti, A. E., \& di Prampero, P. E. (2002). Mechanical efficiency of cycling with a new developed pedal-crank. Journal of Biomechanics, 35(10), 1387-1398. 